\title{
Preoperative detection and management of immune heparin-induced thrombocytopenia in patients undergoing heart surgery with iloprost
}

George M. Palatianos, MD ${ }^{\mathrm{a}}$

Christophoros N. Foroulis, MD

Maria I. Vassili, MD ${ }^{\mathrm{b}}$

Phaedra Matsouka, MD ${ }^{b}$

George M. Astras, MD

George H. Kantidakis, MDa

Eugenia Iliopoulou, $\mathrm{BSc}^{\mathrm{c}}$

Efthimia N. Melissari, MD ${ }^{\mathrm{c}}$

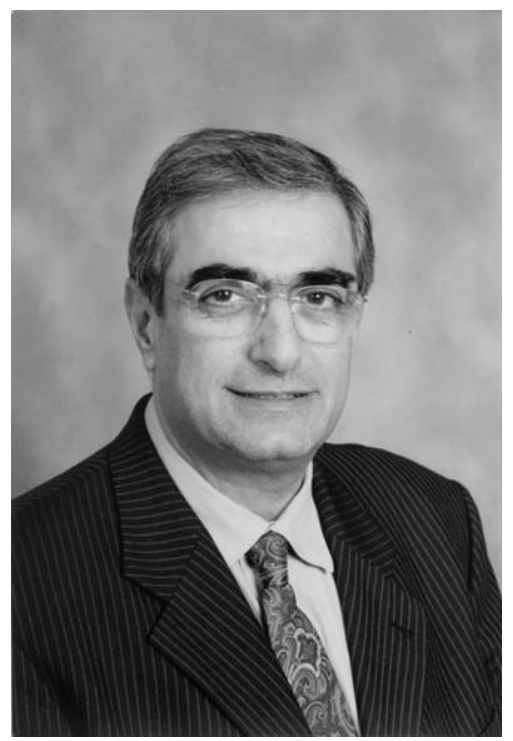

Dr Palatianos

From the Third Department of Cardiac Surgery, ${ }^{\mathrm{a}}$ Department of Anesthesiology, ${ }^{\mathrm{b}}$ and Coagulation and Hemostasis Laboratory, Onassis Cardiac Surgery Center, Athens, Greece.

Read at the Eighty-third Annual Meeting of The American Association for Thoracic Surgery, Boston, Mass, May 4-7, 2003.

Received for publication April 29, 2003; revisions requested Aug 13, 2003; accepted for publication Aug 18, 2003.

Address for reprints: George M. Palatianos, MD, Onassis Cardiac Surgery Center, 356 Sygrou Avenue, 17674 Athens, Greece (E-mail: palatianos@otenet.gr).

J Thorac Cardiovasc Surg 2004;127:548-54 0022-5223/\$30.00

Copyright (C) 2004 by The American Association for Thoracic Surgery

doi:10.1016/j.jtcvs.2003.08.042
Objective: The objective of this study was to evaluate our protocol for the identification and management of patients with immune heparin-induced thrombocytopenia undergoing cardiac surgery.

Methods: Among 1518 patients who underwent cardiac surgery between June 1998 and May 2001, $32(2.1 \%)$ presented with platelet counts less than $150,000 / \mathrm{mm}^{3}$ preoperatively or a history of prolonged ( $>3$ days) intravenous exposure to heparin or both. These 32 patients were evaluated with an enzyme-linked immunosorbent assay for antibodies against heparin-platelet factor 4 complex. Platelets of patients with detected antibodies were tested with the prostacyclin analog iloprost for inhibition of heparin aggregation and determination of the inhibiting concentration and corresponding intravenous infusion rate of iloprost. Patients with antibodies received heparin after complete platelet inhibition with iloprost infusion. Hypotension was prevented or treated with intravenous noradrenaline. Ten randomly selected patients with similar preoperative characteristics, no previous extended exposure to heparin, and normal platelet counts served as controls.

Results: Ten of the 32 patients (group A, 31.3\%) and none of the controls had antibodies against heparin-platelet factor 4 complex. Patients in group A underwent surgery with iloprost $\left(6-24 \mathrm{ng} \cdot \mathrm{kg}^{-1} \cdot \mathrm{min}^{-1}\right)$ and had their blood pressure maintained at greater than $95 \mathrm{~mm} \mathrm{Hg}$ with norepinephrine infusion $\left(1-4 \mu \mathrm{g} \cdot \mathrm{kg}^{-1}\right.$ $\left.\cdot \min ^{-1}\right)$. Operative mortality was zero. There were no thrombotic complications or bleeding requiring exploration. One patient in group A bled $1310 \mathrm{~mL} / 6$ hours but did not need exploration. There was no difference in postoperative blood loss and morbidity between groups. Platelet counts were reduced by $12.5 \% \pm 8.7 \%$ (group A) and $38.1 \% \pm 15.2 \%$ (control) $(P<.001) 1$ hour postoperatively and reached preoperative values by the fifth postoperative day.

Conclusions: Immune heparin-induced thrombocytopenia can be detected preoperatively among patients with a low platelet count or a history of prolonged heparin exposure or both. Cardiac surgery can be safely undertaken using iloprost-induced platelet inhibition during heparinization.

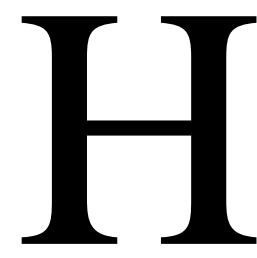

eparin-induced thrombocytopenia and thrombosis (HITT), or heparin-induced thrombocytopenia type II, is an immunologic disorder that affects patients who receive prolonged ( $>3$ days) heparin anticoagulation. ${ }^{1-3}$ When heparin enters the blood, it forms a complex with platelet factor (PF) 4. Patients with HITT have been sensitized against the heparin-PF4 complex, producing mainly immunoglobulin $\mathrm{G}$ antibodies against it. ${ }^{4}$ On reexposure to heparin, these 
antibodies bind to the heparin-PF4 complex at the platelet surface receptor $\mathrm{Fc}$, causing platelet activation, release of procoagulant active microparticles and more PF4, and, consequently, platelet aggregation. The newly released PF4 reacts with the circulating heparin and eventually with the endothelial surface-attached heparin-like molecules, promoting reaction with the specific antibodies. ${ }^{2,3}$ This leads to activation of more platelets and to a vicious cycle that causes platelet aggregation, thromboembolic complications, and bleeding diathesis in association with thrombocytopenia. ${ }^{3,5}$ Patients with HITT antibodies are at high risk $(\sim 50 \%)$ for experiencing thromboembolic complications, bleeding, and sudden death on reexposure to heparin. Death, limb loss, myocardial infarction, coronary artery graft occlusion, mesenteric infarction, deep venous thrombosis, pulmonary embolism, stroke, intracardiac mural thrombosis, bilateral adrenal hemorrhagic infarcts, and bleeding have occurred intraoperatively or early postoperatively in patients with HITT receiving heparin. ${ }^{1,3,5-8}$

The management of patients with HITT antibodies who require cardiac surgery is challenging, because heparin anticoagulation is an integral part of cardiac operations performed with or without extracorporeal circulation. A standard approach to these patients has not yet been established, although several therapeutic options have been proposed on the basis of using anticoagulants other than heparin. ${ }^{1,3}$ However, experience with the Food and Drug Administrationapproved alternative anticoagulants is limited, specific antidotes are not available, and special tests not readily available are required to monitor the effectiveness of the alternatives. $^{3,5,6}$

In an effort to prevent serious perioperative thromboembolic and bleeding complications in our patients, we established a protocol for the identification and management of HITT-sensitive patients who need cardiac surgery with full heparinization. The purpose of this project was 2-fold: the preoperative recognition of patients with heparin-sensitive antiplatelet antibodies and the prevention of platelet activation by temporary platelet function inhibition during heparinization. Our management protocol was based on reports by Addonizio and associates, ${ }^{9,10}$ Kappa and collaborators, ${ }^{11,12}$ and Palatianos and colleagues (on the use of the prostacyclin analog iloprost [ZK 36374]). ${ }^{13}$ Addonizio and associates $^{9,10}$ and Kappa and associates ${ }^{11,12}$ reported platelet preservation with iloprost during cardiopulmonary bypass (CPB) and showed that temporary platelet inhibition with iloprost allowed safe heparin administration in patients with HITT undergoing cardiac or vascular operations.

\section{Methods}

\section{Patients}

From June 1998 to June 2001, 1518 patients underwent cardiac surgery in our department. Of these patients, $32(2.1 \%)$ were found to have thrombocytopenia (platelet counts $<150,000 / \mathrm{mm}^{3}$ ) or a positive history of prolonged ( $>3$ days) exposure to heparin or both. These 32 patients underwent the usual hemostatic assessment and had their bleeding times tested. They were also tested for heparin-induced platelet aggregation (HIPA) using porcine heparin (heparin sodium, Leo Pharmaceutical Products, Ballerup, Denmark) after 14 days from cessation of any aspirin intake. Patients with plasma aggregating normal donor platelets in the presence of heparin (positive HIPA assay) were evaluated with an enzymelinked immunosorbent (ELISA) assay (Diagnostica Stago, Taverny, France) for anti-heparin-PF4 antibodies (HITT antibodies). 2,4,14,15 Patients with HITT antibodies were subsequently tested with a HIPA assay using increasing micromolar concentrations of iloprost (Ilomedin, Berlex Laboratories, Edison, NJ) to determine the dose that completely inhibited platelet aggregation to heparin. ${ }^{9-12}$ During surgery, patients with anti-heparin-PF4 antibodies were treated with iloprost to achieve complete inhibition of HIPA before heparinization (group A or treated group). Ten additional patients with normal platelet counts and without history of prolonged exposure to heparin or thrombosis were matched for age, gender, and procedure, and were included in the study as controls (group C). Our hospital's ethics committee approved the use of iloprost in our patients with HITT antibodies. Each patient signed an informed consent form.

\section{Anesthesia Protocol}

Anesthesia was induced with midazolam $(0.05-0.075 \mathrm{mg} / \mathrm{kg}$ of body weight), etomidate $(0.3 \mathrm{mg} / \mathrm{kg})$, and fentanyl $(10-15 \mu \mathrm{g} / \mathrm{kg})$. Neuromuscular block was induced by intravenous pancuronium or rocuronium $(0.15 \mathrm{mg} / \mathrm{kg})$ and maintained by continuous infusion of cisatracurium $\left(1.5-2.0 \mu \mathrm{g} \cdot \mathrm{kg}^{-1} \cdot \mathrm{min}^{-1}\right)$. Anesthesia was maintained using sevoflurane $0.5 \%$ to $2.0 \%$ in oxygen/air and additional boluses of fentanyl as needed or a continuous infusion of remifentanil $\left(0.2-1.0 \mu \mathrm{g} \cdot \mathrm{kg}^{-1} \cdot \mathrm{min}^{-1}\right)$.

Each patient had a 3-lumen central venous catheter, pulmonary artery catheter, and radial arterial catheter positioned before anesthesia induction. During surgery, there was continuous monitoring of the central venous, pulmonary artery, and arterial pressures; urinary output; electrocardiogram; and nasopharyngeal and rectal temperatures. Tissue oxygenation was monitored with pulse oximetry, and arterial blood gases were frequently tested.

\section{Surgical Protocol and Postoperative Management}

After anesthesia induction, iloprost infusion was started at $3 \mathrm{ng}$. $\mathrm{kg}^{-1} \cdot \min ^{-1}$ intravenously and was increased progressively (doubled) every 5 minutes up to the dose corresponding to the concentration of iloprost, which in vitro inhibited platelet aggregation to heparin. A HIPA test was performed 5 minutes after reaching the target dose. Once complete inhibition of platelet aggregation to heparin was demonstrated, the patients were given porcine heparin for full heparinization (heparin sodium, $300 \mathrm{IU} / \mathrm{kg}$ intravenously). Norepinephrine was infused at $0.05 \mu \mathrm{g} \cdot \mathrm{kg}^{-1} \cdot \mathrm{min}^{-1}$ to prevent or treat hypotension (systolic blood pressure $<100 \mathrm{~mm} \mathrm{Hg}$ ). For cases requiring $\mathrm{CPB}$, the activated coagulation time (ACT) was maintained at more than 480 seconds throughout perfusion. Additional heparin was administered if needed. CPB was established with a roller pump (Stöckert-Shiley Instruments, Munich, Germany), a hollow fiber membrane oxygenator (Quadrox, Jostra, 


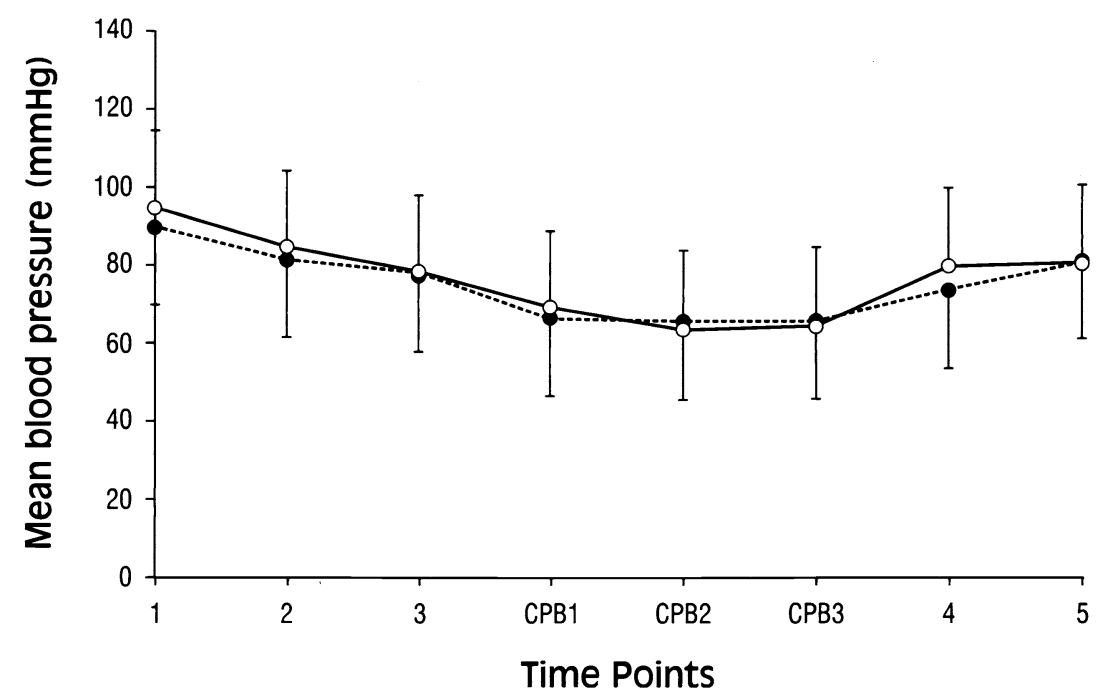

Figure 1. Mean blood pressure changes. Time points: 1, on arrival to the operating room; 2, iloprost infusion begins; 3, before CPB; CPB1, after start of perfusion; CPB2, middle of perfusion; CPB3, before end of perfusion; 4, after CPB; 5, first hour in ICU. Control group (solid line); iloprost-treated group (dashed line).

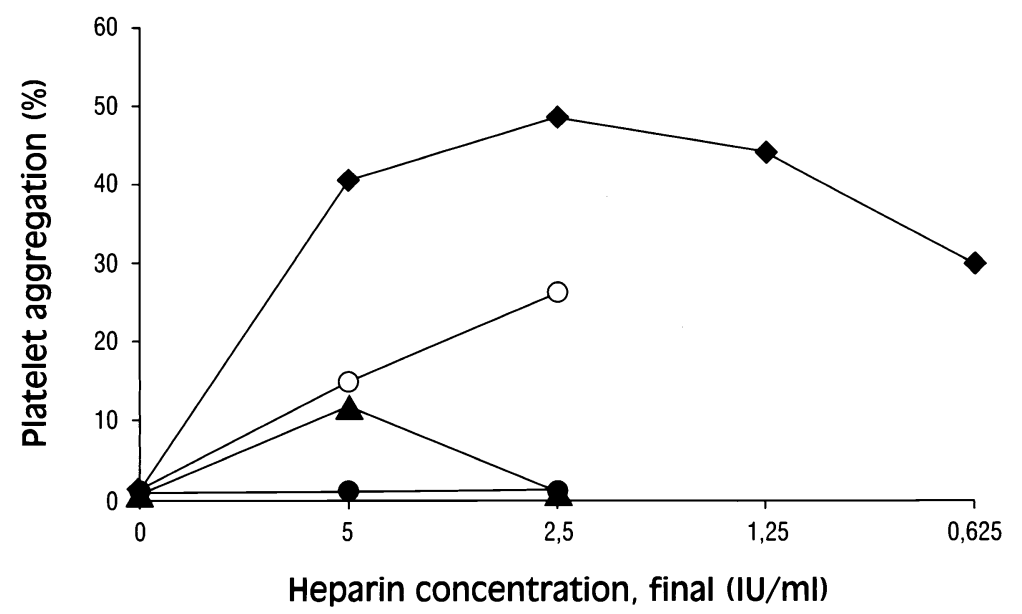

Figure 2. Effect of iloprost at various concentrations and corresponding infusion rates on HIPA in vitro. $\bullet$, No iloprost; $\bigcirc$, iloprost $60 \mathrm{pg} / \mathrm{mL}\left(3 \mathrm{ng} \cdot \mathrm{kg}^{-1} \cdot \mathrm{min}^{-1}\right) ; \Delta, 120 \mathrm{pg} / \mathrm{mL}\left(6 \mathrm{ng} \cdot \mathrm{kg}^{-1} \cdot \mathrm{min}^{-1}\right)$; 0 , iloprost, $240 \mathrm{pg} / \mathrm{mL}(12$ $\left.\mathrm{ng} \cdot \mathbf{k g}^{-1} \cdot \min ^{-1}\right)$.

Hirrlingen, Germany), and an arterial filter (HBF 40, Jostra) at $32^{\circ} \mathrm{C}$ to $33^{\circ} \mathrm{C}$ for cases undergoing coronary revascularization procedures and at $28^{\circ} \mathrm{C}$ for valve surgery cases. Cardioprotection was achieved with induced cardioplegic arrest. Cold $\left(8^{\circ} \mathrm{C}\right)$ blood cardioplegic solution was infused retrograde through the coronary sinus and antegrade through the aortic root. Surface-heparinized extracorporeal circuits were not used in these patients. Off-pump procedures were performed using the Octopus 3 flexible stabilization system (Medtronic, Inc, Minneapolis, Minn) under systemic heparinization (heparin dose 150-200 IU/kg, ACT > 350 seconds). On termination of $\mathrm{CPB}$, or after construction of the bypass grafts for the off-pump coronary bypass cases, protamine sulfate (Leo Pharmaceutical Products) was administered to neutralize the hep- arin and return the ACT to normal values. The patients were transferred to the intensive care unit (ICU), ventilated, continuously monitored for arterial and pulmonary artery pressures and electrocardiogram, and administered iloprost infusion. After demonstration of the absence of circulating heparin with the Hepcon HMS/HMS Plus blood analyzer (Medtronic, Inc), the iloprost infusion was reduced to half and was further halved every 30 minutes until it was discontinued after the first hour in the ICU. Iloprost was used solely for platelet inhibition according to the protocol dose. It was not modified to treat blood pressure changes. These conditions were treated as needed with the standard medications used in the immediate postoperative period. Blood losses from the chest tubes were recorded every hour. 
Postoperative management was performed according to a standard protocol. If there was increased bleeding diathesis, red blood cells, platelets, and fresh frozen plasma were administered as needed to maintain the hematocrit above $26 \%$ in hemodynamically stable patients or to correct prolonged coagulation parameters. No heparin was administered to the patients thereafter. The intravenous lines were flushed with heparin-free saline, and any need for anticoagulation was achieved with acenocoumarin (Sintrom, Novartis-Pharma, Basel, Switzerland). Platelet counts were obtained preoperatively, 1 hour after surgery, and on the first and fifth postoperative days, and were corrected for hemodilution according to hematocrit values.

\section{Statistical Methods}

The analysis of the data collected for the study was performed using the following statistical methods, depending on the nature of the data analyzed:

Longitudinal data (ie, measurements on the same variable taken at consecutive time points for each patient) were evaluated by repeated-measures analysis of variance (RM-ANOVA). All other continuous variables measured at a single time point (eg, duration of ICU stay and amounts of blood loss), aggregates of the longitudinal data, and percent reduction of platelet counts from the preoperative values were evaluated by the $t$ test and its nonparametric equivalent (Wilcoxon rank-sum test). Changes from baseline at each time point of measurement within each group (eg, no intergroup comparisons) were evaluated using the Student paired $t$ test and the Wilcoxon signed-rank test. The association between length of perfusion and platelet count reduction was assessed with Spearman's correlation. In general, during the analysis, the parametric methods were used after testing for normality. If the test rejected the normality assumption, then the nonparametric method was used. The $P$ values reported are accompanied by the type of test they are derived from. All calculations were performed using the SASJ statistical package (SAS Institute, Inc, Cary, NC).

\section{Results}

Ten patients were found to have HITT antibodies $(0.65 \%$ of our total patient population; $31.3 \%$ of the patients presented with thrombocytopenia or history of prolonged heparin therapy). Their preoperative bleeding times were within normal range (6.4 \pm 1.94 minutes). Patient characteristics and surgery data appear in Table 1. Iloprost was infused at 6 to $24 \mathrm{ng} \cdot \mathrm{kg}^{-1} \cdot \mathrm{min}^{-1}$, achieving complete platelet inhibition to heparin. In the control group, mean maximal blood pressure decreased by $26.5 \pm 11.1 \mathrm{~mm} \mathrm{Hg}$ before CPB, whereas in the iloprost-treated group, systolic pressure decreased by $37.0 \pm 10.3 \mathrm{~mm} \mathrm{Hg}$ before CPB $(P=.03$, Wilcoxon test). Blood pressure was easily controlled using norepinephrine infusion or phenylephrine boluses (Figure 1). Two patients with high blood pressure did not require norepinephrine; their blood pressure was readily controlled during iloprost infusion with phenylephrine boluses (100 $\mu \mathrm{g})$. Platelet counts were significantly reduced 1 hour after surgery in both groups $(P<.0001, \mathrm{RM}-\mathrm{ANOVA})$. The control group displayed a greater decrease in platelet count from baseline
TABLE 1. Patient characteristics and operative data

\begin{tabular}{lcc}
\hline & $\begin{array}{c}\text { Treated group } \\
(\mathbf{n}=\mathbf{1 0})\end{array}$ & $\begin{array}{c}\text { Control group } \\
(\mathbf{n}=\mathbf{1 0})\end{array}$ \\
\hline Female gender & 1 & 1 \\
Age (y) & $61.1 \pm 7.2$ & $62.7 \pm 8.4$ \\
Operation performed & & 7 \\
CABG & 7 & 1 \\
MVR & 3 & 1 \\
CABG and MV repair & 0 & 1 \\
CABG and MVR & 0 & $97.1 \pm 59.6$ \\
CPB time (min) & $67.6 \pm 53.0$ & $22,100 \pm 9,868$ \\
Heparin dose (IU) & $16,950 \pm 5,449$ & $160.00 \pm 45.9$ \\
Protamine dose (mg) & $135.00 \pm 51.9$ & $130 \pm 23$ \\
ACT (sec) & & $499 \pm 116$ \\
Baseline & $133 \pm 15$ & $132 \pm 11$ \\
$\quad$ During CPB & $523 \pm 122$ & \\
$\quad$ After protamine & $141 \pm 20$ & \\
\hline
\end{tabular}

The \pm values are SDs. $A C T$, Activated coagulation time; $C A B G$, coronary artery bypass grafting; $C P B$, cardiopulmonary bypass; $M V$, mitral valve; $M V R$, mitral valve replacement.

at 1 hour and 1 day postoperatively than the treated group ( $P$ $<.0001$ and .0487, respectively, RM-ANOVA) (Table 2). Iloprost caused a concentration-dependent inhibition of platelet aggregation preoperatively in vitro (Figure 2). Complete inhibition of platelets occurred with iloprost infusion at 6 to $24 \mathrm{ng} \cdot \mathrm{kg}^{-1} \cdot \mathrm{min}^{-1}$, corresponding to plasma concentrations of 120 to $480 \mathrm{pg} / \mathrm{mL}$. After intravenous iloprost administration, the HIPA test became negative at the corresponding concentrations, as was identified in vitro in 9 patients. In the remaining patient, the iloprost infusion rate had to be doubled during surgery to obtain complete platelet inhibition.

There was no operative mortality. Hematocrit values decreased significantly in both groups over time $(P<.0001$, RM-ANOVA) (Table 2). There was no significant difference between groups overall or in the time progression of the hematocrit value or at any time point measured. In the treated group, 1 patient bled $>1000 \mathrm{~mL}(1310 \mathrm{~mL})$ in the first 6 postoperative hours but did not require exploration; he had prolonged coagulation parameters without free circulating heparin (negative Hepcon test). The bleeding was controlled with fresh frozen plasma, platelet, and red blood cell transfusions. There was no significant difference in mediastinal blood losses in the first 6 postoperative hours between the treated and control groups $(317.5 \pm 355.9 \mathrm{~mL}$ vs $304.5 \pm 126.8 \mathrm{~mL}$, respectively, $P=.3634$, Wilcoxon test) and no difference in transfusion requirements $(P=.67$, Wilcoxon test). A patient in the treated group demonstrated low cardiac output that required the use of an intraaortic balloon pump for 24 hours. Mild transient jaundice appeared in 1 patient in each group. In the control group, 1 patient required prolonged ( $>48$ hours) intubation, and another patient required reoperation with construction of a 
TABLE 2. Changes in hematocrit and platelet counts

\begin{tabular}{|c|c|c|c|}
\hline Parameter & Treated group & Control group & $P$ value (test) \\
\hline \multicolumn{4}{|l|}{ Platelet count $\left(\times 10^{3} / \mathrm{mm}^{3}\right)$} \\
\hline Baseline & $151.6 \pm 63.012$ & $212.7 \pm 52.637$ & NS \\
\hline First hour in ICU & $135.7 \pm 65.488$ & $127.7 \pm 27.949$ & .0001 (RM-ANOVA) \\
\hline First postoperative day & $114.8 \pm 49.409$ & $136.7 \pm 40.472$ & .0487 (RM-ANOVA) \\
\hline On discharge & $165.2 \pm 65.149$ & $198.6 \pm 96.464$ & NS \\
\hline \multicolumn{4}{|l|}{ Hematocrit $(\%)$} \\
\hline Baseline & $42.28 \pm 1.99$ & $41.01 \pm 3.52$ & NS \\
\hline First hour in ICU & $31.07 \pm 4.72$ & $30.3 \pm 4.58$ & NS \\
\hline First postoperative day & $32.08 \pm 3.01$ & $33.33 \pm 3.66$ & NS \\
\hline On discharge & $32.69 \pm 5.50$ & $33.11 \pm 3.85$ & NS \\
\hline
\end{tabular}

RM-ANOVA, Repeated-measures analysis of variance; ICU, intensive care unit; $N S$, not significant.

venous bypass graft because of malfunction of an internal thoracic artery bypass graft. The lengths of postoperative stay in the ICU and postoperative hospitalization were 1.6 \pm 1.2 days and $6.5 \pm 1.9$ days, respectively, without significant difference between groups.

\section{Discussion}

Thrombocytopenia in patients with prior prolonged exposure to heparin or with thrombotic or thromboembolic events under heparin therapy may indicate the presence of HITT. ${ }^{1-4}$ However, this immune disorder may be concealed, and severe thromboembolic events may develop on heparin administration in some patients with normal platelet counts. ${ }^{4}$ Unfortunately, in a large proportion of patients, the condition is recognized after the occurrence of a serious complication. In their 14-year series, Warkentin and Kelton ${ }^{1}$ found that approximately half of HITT-sensitive patients were recognized only after a complicating thrombotic event. A high index of suspicion is mandatory for preoperative recognition of patients with HITT and prevention of devastating complication(s) on heparin administration. The condition may be diagnosed by HIPA and serotonin radioimmunoassay release tests or serologically by ELISA for detection of HITT-specific antibodies against the heparin-PF4 complex. ${ }^{3,4}$

Our protocol addresses the preoperative recognition of HITT-sensitive patients and their intraoperative management for prevention of activation of the HITT process. In this protocol, HITT is diagnosed preoperatively by screening all patients who present with low platelet counts $\left(<150,000 / \mathrm{mm}^{3}\right)$ or a history of prolonged heparin therapy or thrombosis regardless of platelet count. Currently, in our practice, all our patients scheduled for surgery undergo a preadmission complete blood count and platelet count within the frame of a preadmission evaluation. No patient with a low platelet count is scheduled for surgery without prior investigation of his or her thrombocytopenia. In our laboratory, the special diagnostic workup can be completed within 5 to 6 hours. The determination of the iloprost concentration that inhibits HIPA is usually performed within 1 hour before heparinization for CPB. Therefore, with proper mobilization of the involved services, this protocol can be applied to cases requiring urgent surgery. Since we started using this protocol, we have not seen any of the unexpected postoperative hard-to-manage bleeding that we used to face sporadically. In our experience, $31.3 \%$ of patients with thrombocytopenia who presented for cardiac surgery tested positive for HITT antibodies. Patients testing positive for HIPA are further tested with ELISA for HITT antibodies. In patients with HITT antibodies, surgery is postponed for approximately 3 months if their condition allows it. If the antiplatelet antibodies disappear from the patient's plasma, surgery proceeds with standard $\mathrm{CPB}$ and heparin. Any further exposure postoperatively to either unfractionated or low molecular weight heparin is strictly avoided. ${ }^{3,5,16-18}$ In case the patient's condition forbids delay of surgery, we use iloprost for temporary platelet inhibition (as described previously) to prevent HITT-related complications. Again, heparin is not used postoperatively in any form. This protocol allows the safe use of heparin during CPB in patients with HITT antibodies.

Iloprost, a stable analog of prostacyclin with a half-life of 30 minutes, is effective in completely inhibiting platelets by binding to specific platelet receptors, activating adenyl cyclase, and increasing the intracellular cyclic adenosine monophosphate levels. In addition, iloprost acts on the arterial smooth muscle with a vasodilatory effect that may lead to hypotension. ${ }^{19}$ At appropriate infusion rates, iloprost allows safe heparin administration in patients with HITT antibodies provided there is close monitoring of hemodynamics and careful use of alpha agonists (norepinephrine and phenylephrine) to prevent or treat hypotension. ${ }^{9-13}$ Our data support the hypothesis that heparin may be used with impunity in patients with HITT when their platelets are completely inhibited with iloprost and their hemodynamics are closely monitored. The main side effect of iloprost is hypotension secondary to vasodilation. However, in our experience, hypotension during iloprost infusion was 
readily controlled with norepinephrine infusion (1-4 $\mu \mathrm{g}$. $\mathrm{kg}^{-1} \cdot \min ^{-1}$ ) or phenylephrine boluses. In this report, we present our first 10 patients in whom HITT antibodies were detected preoperatively. Our overall hospital experience to date with patients who test positive for HITT is satisfactory. This protocol has been used successfully for the identification and management of these patients so they can undergo cardiac surgery without the thrombotic and hemorrhagic complications related to their sensitivity to heparin.

\section{Conclusion}

HITT should be suspected in every patient with thrombocytopenia or a positive history of prolonged heparin therapy or thrombosis. In such patients, any heparin should be avoided until testing for immune anti-heparin-PF4 antibodies is completed. Immune HITT antibodies can be effectively diagnosed preoperatively, and patients can safely undergo cardiac surgery with temporary iloprost-induced platelet function inhibition during heparinization and cautious blood pressure control with alpha agonists. data.

We thank Yannis Bassiakos, $\mathrm{PhD}$, for statistical analysis of the

\section{References}

1. Warkentin TE, Kelton JG. A 14-year study of heparin-induced thrombocytopenia. Am J Med. 1996;101:502-7.

2. Warkentin TE, Kelton JG. Temporal aspects of heparin-induced thrombocytopenia. $N$ Engl J Med. 2001;344:1286-92.

3. Follis F, Schmidt CA. Cardiopulmonary bypass in patients with heparin-induced thrombocytopenia and thrombosis. Ann Thorac Surg. 2000;70:2173-81.

4. Visentin GP, Ford SE, Scott JP, Aster RH. Antibodies from patients with heparin-induced thrombocytopenia/thrombosis are specific for platelet factor 4 complexed with heparin or bound to endothelial cells. J Clin Invest. 1994;93:81-8.

5. Messmore HL Jr, Wehrmacher WH. Management of heparin-induced thrombocytopenia and postoperative bleeding in cardiovascular surgery. In: Pifarré R, editor. Management of bleeding in cardiovascular surgery. Philadelphia: Hanley \& Belfus; 2000. p. 279-85.

6. Edmunds LH Jr. HIT, HITT, and desulfatohirudin: look before you leap. J Thorac Cardiovasc Surg. 1995;110:1-3.

7. Aouifi A, Blanc P, Piriou V, Bastien OH, French P, Hanss M, et al. Cardiac surgery with cardiopulmonary bypass in patients with HIT II heparin-induced thrombocytopenia. Ann Thorac Surg. 2001;71:67883.

8. Burke AP, Mezzetti T, Farb A, Zech ER, Virmani R. Multiple coronary artery graft occlusion in a fatal case of heparin-induced thrombocytopenia. Chest. 1998;114:1492-9.

9. Addonizio VP Jr, Fisher CA, Jenkin BK, Strauss JF 3rd, Musial JF, Edmunds LH Jr. Iloprost (ZK36374), a stable analogue of prostacyclin, preserves platelets during simulated extracorporeal circulation. J Thorac Cardiovasc Surg. 1985;89:926-33.

10. Addonizio VP, Fisher CA, Kappa JR, Ellison N. Prevention of heparin-induced thrombocytopenia during open heart surgery with iloprost (ZK 36374). Surgery. 1987;102:796-807.

11. Kappa JR, Horn MK, Fisher CA, Cottrell ED, Ellison N, Addonizio VP Jr. Efficacy of iloprost (ZK 36374) versus aspirin in preventing heparin-induced platelet activation during cardiac operations. J Thorac Cardiovasc Surg. 1987;94:405-13.

12. Kappa JR, Fisher CA, Todd B, Stenach N, Bell P, Campell F, et al. Intraoperative management of patients with heparin-induced thrombocytopenia. Ann Thorac Surg. 1990;49:714-23.
13. Palatianos GM, Dewanjee MK, Smith W, Novak S, Hsu LC, Kapadvanjwala $\mathrm{M}$, et al. Platelet preservation during cardiopulmonary bypass with iloprost and Duraflo-II heparin-coated surfaces. ASAIO Trans. 1991;37:620-2.

14. Sheridan D, Carter C, Kelton JG. A diagnostic test for heparin-induced thrombocytopenia. Blood. 1986;67:27-30.

15. Isenhart CE, Brandt JT. Platelet aggregation studies for the diagnosis of heparin-induced thrombocytopenia. Am J Clin Pathol. 1993;99:32430.

16. Cummins D, Halil O, Amin S. Which patients undergoing cardiopulmonary bypass should be assessed for development of heparin-induced thrombocytopenia? Thromb Haemost. 1995;73:890.

17. Warkentin TE, Sheppard JI, Horsewood P, Simpson PJ, Moore JC, Kelton JG. Impact of the patient population on the risk for heparininduced thrombocytopenia. Blood. 2000;96:1703-8.

18. Selleng S, Lubenow N, Wollert HG, Muiiejans B, Greinbacher A. Emergency cardiopulmonary bypass in a bilaterally nephrectomized patient with a history of heparin-induced thrombocytopenia: successful reexposure to heparin. Ann Thorac Surg. 2001;71:1041-2.

19. Krause W, Krais T. Pharmacokinetics and pharmacodynamics of the prostacyclin analogue iloprost in man. Eur J Clin Pharmacol. 1986; 30:61-8.

\section{Discussion}

Dr Michael A. Acker (Philadelphia, Pa). Your method of prospective screening of patients at risk for HIT seems to have identified patients at risk for thrombotic complications and death. In your article you indicate that in elective cases the first thing one should do is probably wait 3 months, because often the antibody levels will decline and often disappear. This becomes a bigger issue when you can't wait or when the antibody levels do not disappear.

You successfully used iloprost in 10 patients identified with HIT, and you followed a similar successful series by Addonizio and colleagues and Kappa and colleagues from the University of Pennsylvania nearly 15 years ago. They found, as you did, that the vasodilatory side effects of iloprost can be reasonably handled in a safe fashion and that iloprost prevents platelet aggregation leading to bleeding and thrombotic complications. Unfortunately, iloprost is not available in the United States.

I have 3 questions, and there are 3 slides. You did not mention the serotonin release assay in your presentation, which is perhaps the oldest and still the most specific and sensitive test for HIT as far as indicating the development of thrombotic complications. You have not used this test. Why?

We found recently that many patients who test positive for heparin antibodies with the ELISA, test negative with the serotonin release assay. We have successfully proceeded with CPB using a 1-time heparin dose without adjuvant or alternative means of anticoagulation in this small group of patients. Do you believe that patients with a negative functional assay such as serotonin or platelet aggregation, yet a positive heparin antibody with the ELISA, define a different group of patients who can be treated safely in the traditional fashion?

Finally, given that iloprost is not available in the United States, and it is interesting to conjecture why not, what is your advice for the U.S. surgeon faced with a patient with HIT? Can you comment on the alternative anticoagulation strategies I have listed, such as alternative prostacyclin analogs (eg, epoprostenol), direct thrombin inhibitors (there are several now), defibrinating agents (eg, ancrod and GPIIb or IIIa receptor inhibitors), or the heparinoids? 
Dr Palatianos. There is no $100 \%$ sensitive assay to identify the patients who will have clinical HITT. We used the ELISA assay because it was available in our hospital. Indeed, there are some patients who test negative and may have clinical HITT, and there are patients who test positive for antibodies and will not have clinical HITT after heparinization for surgery. To improve diagnostic accuracy, we also test platelet aggregation to heparin. The serotonin release assay is a highly sensitive method for detection of HITT antibodies. However, it is technically demanding and not readily available in every hospital. When available, it should be used to identify patients prone to have HITT complications.

Patients who test positive for HITT antibodies with the ELISA test and test negative with the serotonin release assay should be restudied. If the ELISA assay remains positive, I think they should be treated as HITT prone.

Although iloprost is available in Europe, its use for prevention of HITT is not included among its accepted indications. We use it within the frame of the clinical study. There are recent reports promising that newer medications such as thrombin inhibitors or heparin substitutes may become available in the near future. However, a generalized experience with them is lacking.

I think it is important to identify these patients preoperatively. Carefully investigating the patients who come for surgery with low platelet counts or a history of prolonged heparin therapy may help in identifying patients with HITT antibodies. In general, we test the occasionally seen patient with thrombocytopenia with a bleeding time and perhaps with platelet aggregation studies. If these tests are normal, we should not stop there. Some of these patients may have HITT antibodies. With our protocol, we detected HITT antibodies in $31.3 \%$ of these patients. Once we identify the patients, if their condition allows us to wait, postponing surgery is the best thing to do until the antibodies clear, usually within 2 to 3 months. In case surgery cannot be postponed, the patients should be managed with a specific protocol addressing this serious and potentially dangerous problem.

Dr Paschalis Tossios (Cologne, Germany). We know that these patients are sometimes very sick and that they are very difficult to handle when the diagnosis is confirmed preoperatively. In my own experience, heparin-induced thrombocytopenia and associated complications occur more often postoperatively, and they might occur with venous and arterial thromboembolic complications, even with normal platelet counts. Do you have a protocol for when heparin-induced thrombocytopenia is diagnosed during the postoperative course?

Dr Palatianos. Basically, heparin, either unfractionated or low molecular weight, should not be given to these patients postoperatively, not even through a heparin flush to keep arterial or venous lines open. Of course, HITT antibodies may be present with a normal platelet count.

We have not observed any thrombosis in our patients. In case of thrombosis, any heparin preparation that the patient is receiving should be stopped, and some antithrombotic agent could be used. Of course, the site of thrombosis, clinical picture, timing, and general condition of the patient direct the treatment. For this condition, prevention is the best treatment. By making the platelets insensitive to the stimuli with iloprost, we prevent platelet activation and release reaction, aggregation, and thrombosis. 